\title{
BMJ Open Effectiveness of knowledge translation tools addressing multiple high-burden chronic diseases affecting older adults: protocol for a systematic review alongside a realist review
}

\author{
Monika Kastner, ${ }^{1,2}$ Laure Perrier, ${ }^{1}$ Jemila Hamid, ${ }^{1,3}$ Andrea C Tricco, ${ }^{1,2}$ \\ Roberta Cardoso, ${ }^{1}$ Noah M Ivers, ${ }^{4}$ Barbara Liu, ${ }^{5}$ Sharon Marr, ${ }^{6}$ \\ Jayna Holroyd-Leduc, ${ }^{7}$ Geoff Wong, ${ }^{8}$ Lisa Graves, ${ }^{9}$ Sharon E Straus ${ }^{1,10}$
}

To cite: Kastner M, Perrier L, Hamid J, et al. Effectiveness of knowledge translation tools addressing multiple high-burden chronic diseases affecting older adults: protocol for a systematic review alongside a realist review. BMJ Open 2015;5 e007640. doi:10.1136/ bmjopen-2015-007640

- Prepublication history and additional material is available. To view please visit the journal (http://dx.doi.org/ 10.1136/bmjopen-2015007640).

Received 11 January 2015 Accepted 15 January 2015

CrossMark

For numbered affiliations see end of article.

Correspondence to Dr Monika Kastner; monika.kastner@utoronto.ca

\section{ABSTRACT}

Introduction: The burden of chronic disease is a global phenomenon, particularly among people aged 65 years and older. More than half of older adults have more than one chronic disease and their care is not optimal. Chronic disease management (CDM) tools have the potential to meet this challenge but they are primarily focused on a single disease, which fails to address the growing number of seniors with multiple chronic conditions.

Methods and analysis: We will conduct a systematic review alongside a realist review to identify effective CDM tools that integrate one or more highburden chronic diseases affecting older adults and to better understand for whom, under what circumstances, how and why they produce their outcomes. We will search MEDLINE, EMBASE, CINAHL, AgeLine and the Cochrane Library for experimental, quasi-experimental, observational and qualitative studies in any language investigating CDM tools that facilitate optimal disease management in one or more high-burden chronic diseases affecting adults aged $\geq 65$ years. Study selection will involve calibration of reviewers to ensure reliability of screening and duplicate assessment of articles. Data abstraction and risk of bias assessment will also be performed independently. Analysis will include descriptive summaries of study and appraisal characteristics, effectiveness of each CDM tool (metaanalysis if appropriate); and a realist programme theory will be developed and refined to explain the outcome patterns within the included studies.

Ethics and dissemination: Ethics approval is not required for this study. We anticipate that our findings, pertaining to gaps in care across highburden chronic diseases affecting seniors and highlighting specific areas that may require more research, will be of interest to a wide range of knowledge users and stakeholders. We will publish and present our findings widely, and also plan more active dissemination strategies such as workshops with our key stakeholders.

\section{Strengths and limitations of this study}

- Our systematic review will be the first to elucidate a more in-depth understanding of chronic disease management across many common, high-burden chronic diseases affecting older adults: our systematic review will inform which chronic disease management (CDM) tools work (or not) for targeted conditions and which of their components have the most potential for impact to address the complex health needs of seniors; and the realist review will inform programme theories that explain how, for whom, under what circumstances and why CDM tools work.

- There are few examples of a realist review conducted alongside a systematic review and there may be a benefit to this in terms of efficiency of conduct, so our investigation will contribute to advancing knowledge of this method.

- Our search strategy is expansive, but there is a potential that we may not capture all existing CDM tools.

Trial registration number: Our protocol is registered with PROSPERO (registration number CRD42014014489).

\section{INTRODUCTION}

The burden of chronic disease is a global phenomenon, particularly among peopled aged 65 years and older. Worldwide projections indicate that by 2050, two billion people will be aged 60 years and older. ${ }^{1-3}$ Older adults are living longer than previous generations, so they are at increased risk for developing multiple chronic diseases, which is expected to pose a significant economic 
burden worldwide. ${ }^{2-7}$ Currently, in Canada, $10 \%$ of seniors with the most complex health needs account for $60 \%$ of total annual healthcare spending. ${ }^{8}{ }^{9}$ If we do not address the delivery of healthcare services, the increasing number of seniors is projected to cost $\$ 24$ billion more annually (50\% more than today) in Canada. ${ }^{8} 9$ Further adding to this challenge, more than half of older adults have more than one chronic disease ${ }^{10} 11$ and their care is not optimal, with only $55 \%$ receiving appropriate care. ${ }^{12}{ }^{13}$ Chronic disease management (CDM) tools (ie, tools that facilitate ongoing, proactive and preventative support for optimal disease management) are potential strategies to meet this challenge, but they are not usually developed for seniors with multiple chronic diseases or created for sustained use. Such tools are primarily focused on a single disease $;{ }^{14}{ }^{15}$ this fails to address the complex needs of seniors with multiple chronic conditions.

Evidence is limited on the care of people with multiple chronic conditions. A systematic review by Smith et $a l^{16}$ investigated the effectiveness of interventions in patients with multiple comorbidities. However, this review did not investigate why and under what circumstances interventions addressing multiple chronic conditions are effective or not (and considered only those that were tested in primary care and community settings), and did not search for any CDM tool, quality improvement strategy or knowledge translation (KT) intervention across diseases (collectively referred hereon as CDM tools). To address the needs of seniors with multiple chronic diseases, we need to better understand which CDM tools are effective across specific high-burden chronic diseases affecting seniors, and which components of these interventions optimise their impact, how, for whom, under what circumstances and why.

We aim to synthesise the literature to identify effective $\mathrm{CDM}$ tools that integrate one or more high-burden chronic diseases affecting people aged $\geq 65$ years. We will also conduct a realist synthesis alongside our systematic review to explore what about CDM tools work, for whom, under what contexts, how and why. ${ }^{17}$

\section{METHODS AND ANALYSIS \\ Study design}

We will conduct a systematic review alongside a realist review. Realist synthesis is particularly relevant for making sense of context sensitive complex interventions with a heterogeneous evidence base where traditional systematic reviews would often conclude that there is limited or no evidence to inform next steps. ${ }^{18}$ The conduct of realist reviews is conducive to the study of complex interventions, as simply 'knowing' what works reveals very little about the mechanisms that cause desired outcomes and the contexts under which they occur, and can lead to assertions that 'nothing works' or 'results are inconsistent'. ${ }^{19} 20$ The reporting of our reviews will be guided by the PRISMA ${ }^{21}$ and RAMESES ${ }^{22}$ criteria. Our protocol was conceived, developed and reviewed by all members of our team, and is registered with PROSPERO, an international register of systematic review protocols (registration number CRD42014014489; http://www.crd.york.ac.uk/PROSPERO) ${ }^{23}$

\section{Eligibility criteria}

We developed our eligibility criteria from our research questions: (1) What is the effectiveness of chronic disease management (CDM) tools addressing one or more high-burden chronic diseases affecting people aged $\geq 65$ years? (2) Can the impact of such tools be optimized? For desired outcomes, what are the causal mechanisms and related triggering contexts? We used the following PICOS ${ }^{24}$ elements to build our eligibility criteria (see online supplementary appendix 1):

\section{Population}

Adults aged $\geq 65$ years. We focused our population to elderly patients, as their CDM needs are complex, understudied and may be different from the needs of those younger than 65 years of age.

\section{Intervention}

CDM tools that facilitate ongoing, proactive and preventative support for optimal disease management in one or more high-burden chronic diseases affecting seniors include one or more quality improvement components (eg, care co-ordination, patient self-management, reminders, education, decision support); are targeted to any healthcare professional, patient and/or caregiver; and are delivered in any format (paper-based, electronic, in-person). We define high-burden chronic diseases affecting seniors as suggested by the Public Health Agency of Canada,${ }^{25}$ the National Institute on Aging of the US Department of Health and Human Services, ${ }^{26}$ and the WHO. ${ }^{2}{ }^{27}$ We will categorise these as: (1) cardiovascular: for example, congestive heart failure, coronary artery disease, atrial fibrillation; (2) metabolic: for example, diabetes; (3) neurological: for example, stroke, dementia; (4) respiratory: for example, chronic obstructive pulmonary diseases; (5) mental health: for example, depression; (6) musculoskeletal: for example, osteoporosis, arthritis; and (7) other chronic disease: for example, urinary incontinence.

\section{Comparator}

Other CDM tools or any control intervention or usual care.

\section{Outcomes-systematic review}

Patient level: Impact of CDM tools for improving diseasespecific CDM as reported by primary studies. For example, if the CDM tool targets improving glycaemic control as part of diabetes care, we would consider glycosylated haemoglobin or haemoglobin A1c level as the primary outcome of interest or any reported composite outcome such as a CDM score. Secondary outcomes will include quality of life, functional status (including 
cognitive, physical, social and psychological functioning), and adherence to treatment and treatment harms (eg, hypoglycaemia for diabetes). Since chronic disease affects men and women differently, ${ }^{28}$ we will also assess all outcomes by sex. Provider level: Initiation of disease management activities according to guideline-informed evidence (eg, diagnostic or laboratory investigations, prescription of medications). Process level: Feasibility and usability of the CDM tool reported in studies. System level: Hospital admission, admission to long-term care, physician and emergency department visits, and costs.

\section{Outcomes-realist review}

The main product of the realist review will be (if possible) an overall realist programme theory that explains the finding of our effectiveness systematic review. Realist reviews typically begin with an 'initial rough' programme theory, which serves as a basic idea about what an intervention is comprised of, how and why it is expected to work and what outcomes it might generate. ${ }^{17}$ This will then allow us to identify and better understand specific ' Context -Mechanism-Outcome Configurations' (CMOCs) for each of the outcomes contained within the programme theory. Explanatory theory is then used to explain the CMOCs found within the programme theory.

We will develop an initial 'rough' programme theory of CDM tools describing the relationships between the stages necessary to reach the final desired outcome: improved health outcomes for patients with multimorbidity. We will do this iteratively through consultations with experts among our team and from the data within our included sources and from any necessary additional searches. For each stage within the programme theory, inferences will be made about what the possible realist explanation might be-that is, for the outcome within a stage, what might the causal mechanism(s) possibly be and under which contexts might they possibly be triggered. Such an analysis will enable us to address our second set of research questions (ie, How may the impact of CDM tools targeting one or more high-burden chronic disease be optimized? For desired outcomes what are the causal mechanisms and related triggering contexts). For example, if one of the systematic review findings was the reduction in HbAlc levels in seniors who completed a 6-month diabetes self-management CDM tool, we will seek explanations of what has caused this outcome to occur (ie, the mechanism(s)) and the contexts in which this happened. We anticipate that within such an explanation, there may be more than one stage needed to achieve the final desired outcome-that is, better diabetes control. For each of these stages, we will identify the 'intermediate' outcome and from the data within the included sources, elucidate what the mechanism(s) might be for the outcome for this stage and its associated triggering context(s). In essence, we will derive CMOCs for each stage within our explanation of how better diabetes control was achieved-that is, produce a realist programme theory for diabetes CDM tools.
Programme theories will be developed for other CDM tools and we will identify if there are commonalities (eg, in the stages needed to achieve desired outcomes) that would enable us to abstract further and construct an overall refined programme theory that explains the finding of our effectiveness systematic review.

\section{Study design}

Experimental (randomised controlled trials (RCTs), quasi-RCTs, non-RCTs), quasi-experimental (interrupted time series, controlled before and after studies), observational (cohort and cross-sectional studies), and qualitative and mixed-methods studies, will be eligible. We are including observational studies because complex interventions are seldom evaluated in RCTs. We will extend our search to also include qualitative and mixedmethods studies as these may potentially include relevant data for programme theory development. To refine our programme theory of our realist investigation, we may also need to iteratively seek additional literature (eg, through expert-identified searching and snowball sampling $\left.{ }^{17}\right)$. We will include studies that meet our criteria for relevance (ie, does the study address our question?) and study quality (see below). Systematic reviews will be identified, but used only to scan their included studies for potentially relevant articles. For the systematic review, we will exclude case-control studies; case reports and opinion-driven reports (editorials, letters and nonsystematic or literature/narrative reviews). We will, however, note the presence of these sources as we may have to return to them to seek out relevant data for programme theory refinement as part of the realist review.

\section{Search strategy}

We will develop a single search strategy for the systematic review and realist review. With the help of an information specialist, we will search MEDLINE, EMBASE, CINAHL, AgeLine and the Cochrane Library in any language. We are restricting our search to 1990 and onwards as evidence indicates that few multimorbidity studies have been published prior to this, ${ }^{16}$ and CDM has substantially changed over the past 15 years. We have completed our search in MEDLINE from 1990 to January 2015 (see online supplementary appendix 2). To help identify studies of older adults aged $\geq 65$ years, we will apply a validated age-specific search filter. ${ }^{29}$ We will also search the grey or difficult to locate literature (ie, conference proceedings, Google Scholar and websites of relevant chronic disease organisations); and scan reference lists of included studies. We will also use the Canadian Agency for Drugs and Technologies in Health (CADTH) grey matter approach, which is a 'deep-web search tool for evidence-based medicine'. ${ }^{30}$ This approach uses a checklist to identify international health technology assessment websites, clinical trial registries and health economics resources. ${ }^{30} \mathrm{~A}$ second information specialist will validate our search strategy using the peer review process of the PRESS checklist. $^{31}$ A preliminary search strategy 
conducted in MEDLINE is available in the online supplementary appendix 2. Once our search strategy is peer reviewed and finalised in MEDLINE, we will adjust and develop this for our other data sources (EMBASE, CINAHL, the Cochrane Library and AgeLine). If during programme theory development and refinement for the realist review we find that we need additional information, we will consult with our information specialist to develop and refine additional searches.

\section{Study selection}

We will perform a calibration exercise among reviewers to ensure reliability of screening of titles and abstracts. This will involve two reviewers independently screening $10 \%$ of a random sample of citations using our online Synthesi.SR Tool (proprietary online systematic review software developed for our Knowledge Synthesis Center at St Michael's Hospital). We will calculate inter-rater agreement using per cent agreement. We will repeat this exercise until we reach a high level of consistency (at least $90 \%$ raw agreement), at which point two reviewers will independently screen titles and abstracts of potentially relevant articles in duplicate (level 1 screening). We will follow a similar calibration procedure to identify potentially relevant articles during level 2 screening (ie, full-text articles). Disagreements at both levels of screening will be resolved through discussions with the research team.

\section{Data collection process}

We will develop a data abstraction form and test it with our reviewers on a random sample of $10 \%$ of included articles. Once reviewers attain at least $90 \%$ raw agreement, two reviewers will independently abstract data on study characteristics, population, setting, CDM tool and its components, outcomes, follow-up, analysis methods, findings and study quality. For the realist review, we will seek data from included sources to iteratively test and refine each section of our initial programme theory. Hence, the data that we need to extract will be informed by our programme theory. For any outcome within a stage of the programme theory, we will seek data that enable us to make inferences about what the mechanism(s) might be and the contexts under which they are triggered. Specifically, sections of text from included sources that support any interpretations we make about the meaning behind the data will be extracted. In other words, if when reading an included source we interpret that a section of text refers to context that is relevant for programme theory development, we will extract that section of text (as well as note its source). Two reviewers will independently map out the relationship between any CMOCs we develop to generate a causative explanation pertaining to the data from the systematic review ${ }^{17}$ (using NVivo V.10.0 to aid in this process); discrepancies will be resolved through team consensus.

\section{Methodological quality assessment}

Study quality will be independently assessed by two reviewers according to study type: the Cochrane Risk of
Bias tool for RCTs; ${ }^{32}$ the Cochrane Effective Practice and Organization of Care (EPOC) tool for non-RCTs, quasi-RCTs (ie, interrupted time series, before-after studies) $;^{33}$ the Newcastle-Ottawa Scale for cohort studies $^{34}$ and the Critical Appraisal Skills Program (CASP) tool for qualitative studies. ${ }^{35}$ Additionally, we anticipate that many tools for CDM will be complex interventions (ie, multifaceted with multiple targets), so we will explore their elements to determine which aspect contributes to its impact. To do this, we will extract information about the overall CDM tool or intervention, as well as its specific components or elements (eg, decision support for clinicians, reminders and education for clinicians and patients) using the Template for Intervention Description and Replication (TIDIER) checklist. ${ }^{36}$ This includes information about the rationale or goal of the elements essential to the intervention; what materials were used in its delivery; who delivered the intervention, and how, where, when and how much; and how well the intervention was delivered as planned. ${ }^{36}$

\section{Data synthesis}

We will perform descriptive summaries of study and appraisal characteristics; and assess the effects of each CDM tools descriptively (eg, data distributions, frequencies, percentages, means, medians, SDs and IQRs). If appropriate, we will perform a meta-analysis to estimate the pooled relative risk (dichotomous outcomes) or mean difference or standardised mean difference (continuous outcomes). Analysis will be performed using the $\mathrm{R}$ statistical software, and the results will be presented using forest plots. We will also perform a synthesis of cost data. We will explore the potential sources of statistical, methodological and clinical heterogeneity. Statistical heterogeneity will be assessed using the $\mathrm{I}^{2}$ statistic. ${ }^{37}$ We will consider pooling if heterogeneity among studies is low to moderate $\left(\mathrm{I}^{2}<25-50 \%\right),{ }^{37}$ and a random effects model will be used to account for the observed heterogeneity. We will perform subgroup analyses: by disease, age (65-75; 76-84; 85+), gender, and CDM tools with similar components or similar combinations of components (eg, education+reminder+feedback) and targets (eg, providers, patients). If data are available, we will perform metaregression analyses to formally test if evidence exists for different effects in different subgroups; and assess publication bias using the Egger test. ${ }^{38}$

CDM interventions and tools are complex (ie, multifaceted with multiple targets), so we will explore their individual elements to determine which aspect contributes to their impact. We will use content analysis to do this: two investigators will review the description of each $\mathrm{CDM}$ tool, and independently document its components, by whom and to which target it was delivered (eg, nurse delivers education to patients), at what frequency (eg, twice a week) and duration (eg, 6 months) this was done, and where or in what context (eg, primary care 
clinic). We will also consider the synthesis of our data according to Wagner's Chronic Care Model (CCM). ${ }^{39} 40$ We will use the CCM to map interventions and their components according to its six organisational/practice change elements for improvement: Healthcare organisation, community resources, self-management support, delivery system design, decision support and clinical information system. ${ }^{39}$ We may also perform additional and more targeted content analysis of interventions (and their components) identified by our stakeholder team as having potential to inform practice (eg, identified as effective and feasible to implement). As a final step, we will interpret the findings and outline the broader implications for practice and future study.

\section{Source selection, analysis and synthesis-realist review}

The review processes for the realist review will be undertaken by two reviewers through regular meetings with the project team, where progress on programme theory development and refinement will be shared and discussed. To assess relevance, the full text of sources included in the systematic review will be read and the following questions asked of the source: ${ }^{41}$

A. Does this source contain any data that could be interpreted as relevant context, mechanism or outcome for programme theory development?

B. What is the CMOC for this relevant data? In other words, if a section of text is describing relevant context, what might the mechanism be and what outcome does it relate to? Any single source might not contain all the information needed to construct the CMOC. Often sources contain mainly data on context and an outcome and little (if any) details on the mechanism. Thus, from any one source it is often only possible to construct a partial CMOC.

C. How does the (full or partial) CMOC relate to the programme theory? Is there any data in this source to indicate how the CMOC relates to the programme theory? In light of this CMOC and any data on the relationship between this CMOC and the programme theory, are any changes needed in the programme theory? If so, how?

D. Finally and related to (B) and (C) above: How trustworthy are the data used to construct the CMOC? Are they rigorous enough to justify any changes to a CMOC? How trustworthy are the data used to refine the relationships within the programme theory? Are they rigorous enough to justify any changes to the programme theory?

It is at this stage that Pawson's concept of rigour is used. ${ }^{41}$ For example, a CMOC based on the opinions expressed in an editorial may be relevant and constructed. The editorial may have a few references, but ultimately it is just the opinion of the authors. The contents of the editorial may be relevant to a CMOC and the programme theory, but caution would be needed before any changes are made to the programme theory to reflect what is in the CMOC until more data of a more rigorous nature are found.

Within a realist review during source selection, extraction, analysis and synthesis, the reviewer is constantly moving between data, to CMOCs and programme theory-that is, moving up and down levels of abstraction. Also, the most common issue that a reviewer will encounter is, any one source often only provides partial 'bits' of relevant information to inform (A) to (D) above. The consequence is, to make up the complete 'picture', bits from more than one source are frequently needed.

\section{DISCUSSION AND DISSEMINATION}

The main objective of this systematic review alongside a realist review is to identify effective CDM tools that integrate one or more high-burden chronic diseases affecting seniors, and to understand the mechanisms underpinning their effectiveness. Our systematic review will inform a more complete understanding of CDM across identified high-burden chronic diseases affecting seniors, and identify effective CDM tools and their components having the most potential for impact to address the complex needs of seniors. There may also be a benefit to conducting a realist review alongside a systematic review to simplify and streamline their conduct compared with conducting them individually. There is currently no published example of this, but there is at least one investigation underway, ${ }^{42}$ so our investigation will also contribute to advancing knowledge of this method.

Our systematic review will inform which CDM tools work (or not) for targeted conditions, and the realist review will inform programme theories that explain how and why CDM tools work. Anticipated outputs of our systematic review include a taxonomy of CDM tools and their components by each chronic disease, whether the tool was designed to target single or multiple chronic conditions, and an understanding of the causal processes and influences on the impact of CDM tools (ie, facilitator and barrier factors, and the mechanisms and contexts underpinning these factors, by whom, and for which targets and settings they are delivered). Additionally, our examination of a wide range of study designs (including observational and qualitative studies) will also contribute to a more in-depth understanding of CDM tools.

Our systematic review will be the first to elucidate a more in-depth understanding of CDM across many common, high-burden chronic diseases affecting older adults. Our work will also contribute to health outcomes by addressing the impact of CDM tools for improving disease-specific CDM and quality of life across a wide range of high-burden chronic conditions. As such, we anticipate that our findings will be of interest to a wide range of knowledge users, including clinicians, seniors and their caregivers, health administrators, educators and KT or implementation science 
researchers. Given the rapidly ageing population worldwide, findings of this review will also be of interest to policymakers and funders. Our work will inform these stakeholders of the gaps and management strategies in care for specific high-burden chronic diseases affecting seniors, and highlight specific areas that may require more research, future funding and allocation of resources.

We will use different KT strategies to ensure that findings from this systematic review are broadly disseminated to the right audiences. These will include publications in open-access, peer-reviewed journals and public websites, presenting our work at relevant geriatric and disease-specific conferences, and producing lay publications of our findings. As part of a more active KT strategy, we will also plan a workshop with our key stakeholders (ie, clinicians, researchers, decision makers and people with multiple chronic diseases) to discuss the findings, generate key messages most relevant to each, and discuss the next steps including the development of a multi-CDM tool that will address current gaps in care for seniors with multiple chronic diseases.

\section{Author affiliations \\ ${ }^{1}$ Li KaShing Knowledge Institute, St Michael's Hospital, Toronto, Ontario, Canada \\ ${ }^{2}$ Division of Epidemiology, Dalla Lana School of Public Health, University of Toronto, Toronto, Ontario, Canada \\ ${ }^{3}$ Department of Clinical Epidemiology and Biostatistics, McMaster University, Hamilton, Ontario, Canada \\ ${ }^{4}$ Department of Family Medicine, Women's College Hospital—University of Toronto, Toronto, Ontario, Canada \\ ${ }^{5}$ Regional Geriatric Program of Toronto; Sunnybrook Health Sciences, Geriatric Medicine, Toronto, Ontario, Canada \\ ${ }^{6}$ Division of Geriatric Medicine McMaster University, St Peter's Hospital/ Hamilton Health Sciences, Hamilton, Ontario, Canada \\ ${ }^{7}$ Department of Medicine and Community Health Sciences, University of Calgary, Calgary, Alberta, Canada \\ ${ }^{8}$ Centre for Primary Care and Public Health, Queen Mary, University of London, London, UK \\ ${ }^{9}$ Department of Family and Community Medicine, St Michael's Hospital, Toronto, Ontario, Canada \\ ${ }^{10}$ Department of Medicine, University of Toronto, St Michael's Hospital, Toronto, Ontario, Canada}

Contributors MK conceived the study. MK, JH, ACT, GW, NMI and SES developed the study design and all authors contributed to the drafting of the protocol. LP developed the search strategy. All authors edited the draft protocol and read and approved the final manuscript.

Funding This research was supported by an Ontario, Canada Ministry of Health and Long-term Care (MOHLTC) Health Systems Research Fund (HSRF) Capacity Award (grant number 06688). ACT is funded by a Canadian Institutes of Health Research New Investigator Award in Knowledge Synthesis. $\mathrm{NMI}$ is funded by a Canadian Institutes of Health Research New Investigator Award in Community Based Primary Health Care and by a New Investigator Award from the Department of Family and Community Medicine, University of Toronto. SES is funded by a Tier 1 Canada Research Chair in Knowledge Translation.

Competing interests None.

Provenance and peer review Not commissioned; externally peer reviewed.

Data sharing statement Unpublished study data such as the search strategies for the other databases (EMBASE, CINAHL, The Cochrane Library and AgeLine) are available on request to the corresponding author.
Open Access This is an Open Access article distributed in accordance with the Creative Commons Attribution Non Commercial (CC BY-NC 4.0) license, which permits others to distribute, remix, adapt, build upon this work noncommercially, and license their derivative works on different terms, provided the original work is properly cited and the use is non-commercial. See: http:// creativecommons.org/licenses/by-nc/4.0/

\section{REFERENCES}

1. Yach D, Hawkes C, Gould CL, et al. The global burden of chronic diseases: overcoming impediments to prevention and control. JAMA 2004;291:2616-22.

2. World Health Organization. Ageing and life course. http://www.who. int/features/factfiles/ageing/en/ (accessed 28 Aug 2014)

3. World Health Organization. Tackling chronic disease in Europe: strategies, interventions and challenges. 2010. http://www.euro.who. int/_data/assets/pdf_file/0008/96632/E93736.pdf (accessed 1 Sep 2014).

4. United Nations Economic and Social Affairs. World population aging. 2013. http://www.un.org/en/development/desa/population/ publications/pdf/ageing/WorldPopulationAgeing2013.pdf (accessed 20 Oct 2014).

5. Global Health and Ageing. Assessing the costs of aging and health care. http://www.nia.nih.gov/sites/default/files/global_health_and_ aging.pdf (accessed 28 Aug 2014)

6. Lee R, Mason A. Costs of aging: the price of maturity. Finance Dev 2011:1-11. http://www.imf.org/external/pubs/ft/fandd/2011/06/pdf/lee. pdf (accessed Oct 2014).

7. The Canadian population: Age and Sex 2011. Census 2011. http:// www12.statcan.gc.ca/census-recensement/2011/as-sa/98-311-x/ 98-311-x2011001-eng.pdf (accessed Oct 2013).

8. Independence, Activity and Good Health Ontario's Action Plan for Seniors. ON Seniors' Secretariat. https://dr6j45jk9xcmk.cloudfront. net/documents/215/ontarioseniorsactionplan-en-20130204.pdf (accessed in Oct 2013).

9. Barnett K, Mercer SW, Norbury M, et al. Epidemiology of multimorbidity and implications for health care, research, and medical education: a cross-sectional study. Lancet 2012;380:37-43.

10. Moore EG, Rosenberg MW, Fitzgibbon SH. Activity limitation and chronic conditions in Canada's elderly, 1986-2011. Disabil Rehabil 1999;21:196-210.

11. Ward BW, Schiller JS. Prevalence of multiple chronic conditions among US adults: estimates from the National Health Interview Survey 2010. Prev Chronic Dis 2013;10:120203.

12. Wenger NS, Solomon $\mathrm{DH}$, Roth $\mathrm{CP}$, et al. The quality of medical care provided to vulnerable community-dwelling older patients. Ann Intern Med 2003;139:740-7.

13. McGlynn EA, Asch SM, Adams J, et al. The quality of health care delivered to adults in the United States. NEJM 2003;348:2635-45.

14. Coleman K, Austin BT, Brach C, et al. Evidence on the chronic care model in the new millennium. Health Aff (Millwood) 2009;28:75-85.

15. Fortin M, Bravo G, Hudon C, et al. Prevalence of multimorbidity among adults seen in family practice. Ann Fam Med 2005;3:223-8.

16. Smith SM, Soubhi H, Fortin M, et al. Managing patients with multimorbidity: systematic review of interventions in primary care and community settings. BMJ 2012;345:e5205.

17. Pawson R, Greenhalgh T, Harvey G, et al. Realist review-a new method of systematic review designed for complex policy interventions. J Health Serv Res 2005;19(Suppl 1):S21-34.

18. Greenhalgh T, Peacock R. Effectiveness and efficiency of search methods in systematic reviews of complex evidence: audit of primary sources. BMJ 2005;331:1064-5.

19. Berwick DM. The science of improvement. JAMA 2008;299:1182-4.

20. Pawson R. The science of evaluation: a realist manifesto. London, UK: Sage Publications Ltd., 2013.

21. Liberati A, Altman DG, Tetzlaff J, et al. The PRISMA statement for reporting systematic reviews and meta-analyses of studies that evaluate healthcare interventions: explanation and elaboration. $B M J$ 2009;339:b2700.

22. Wong $\mathrm{G}$, Greenhalgh $\mathrm{T}$, Westhorp $\mathrm{G}$, et al. RAMESES publication standards: realist syntheses. BMC Med 2013;11:21.

23. PROSPERO: International prospective register of systematic reviews, University of York, United Kingdom. http://www.crd.york.ac. uk/PROSPERO/ (accessed on 1 Sep 2014).

24. Stone PW. Popping the (PICO) question in research and evidence-based practice. Appl Nurs Res 2002;15:197-8.

25. Public Health Agency of Canada. Preventing chronic disease strategic plan 2013-2016. http://www.phac-aspc.gc.ca/cd-mc/ diabetes-diabete/strategy_plan-plan_strategique-eng.php 
26. National Institute on Aging. Living Long \& Well. http://www.nia.nih. gov/sites/default/files/strategic_plan108.pdf

27. World Health Organization. Global brief for World Health Day. 2012. http://whqlibdoc.who.int/hq/2012/WHO_DCO_WHD_2012.2_eng. pdf?ua $=1$ (accessed Oct 2014).

28. Bierman AS, Ahmad F, Angus J, et al. Burden of Illness: Chapter 3. Ontario Women's Health Equity Report. http://powerstudy.ca/wpcontent/uploads/downloads/2013/01/Chapter3-Burdenoflllness.pdf (accessed Jan 2015)

29. Kastner M, Wilczynski NL, Walker-Dilks C, et al. Age-specific search strategies for Medline. J Med Internet Res 2006;25:e25.

30. Canadian Agency for Drugs and Technologies in Health (CADTH) Grey Matters: a practical search tool for evidence-based medicine. http://www.cadth.ca/en/resources/finding-evidence-is/grey-matters (accessed Oct2014)

31. Sampson M, McGowan J, Cogo E, et al. An evidence-based practice guideline for the peer review of electronic search strategies. $J$ Clin Epidemiol 2009;62:944-52.

32. Higgins JP, Altman DG, Gotzsche PC, et al. The Cochrane Collaboration's tool for assessing risk of bias in randomised trials. BMJ 2011:343:d5928.

33. Cochrane Effective Practice and Organization of Care (EPOC) group. EPOC resources: EPOC Risk of Bias for non-randomized controlled trials (NRCTs) and controlled before-after (CBA) studies. http://epoc.cochrane.org/epoc-resources (accessed Mar 2014).

34. Wells GA, Shea B, O'Connell D, et al. The Newcastle-Ottawa Scale (NOS) for assessing the quality of nonrandomised studies in meta- analyses. http://www.ohri.ca/programs/clinical_epidemiology/oxford. asp (accessed Jan 2015)

35. Critical Appraisal Skills Program (CASP) tool: 10 questions for you to make sense out of qualitative research. http://media.wix.com/ugd/ dded87_951541699e9edc71ce66c9bac4734c69.pdf (accessed 1 Sep 2014).

36. Template for Intervention Description and Replication (TIDIER) checklist. http://www.equator-network.org/wp-content/ uploads/2014/03/TIDieR-Checklist-PDF.pdf (accessed Oct 2014).

37. Higgins JP, Thompson SG. Quantifying heterogeneity in a meta-analysis. Stat Med 2002;21:1539-58.

38. Egger M, Davey Smith G, Schneider M, et al. Bias in meta-analysis detected by a simple, graphical test. BMJ 1997;315:629.

39. Wagner EH, Austin BT, Davis C, et al. Improving chronic illness care: translating evidence into action. Health Aff (Millwood) 2001;20:64-78.

40. Barr VJ, Robinson S, Marin-Link B, et al. The expanded Chronic Care Model: an integration of concepts and strategies from population health promotion and the Chronic Care Model. Hosp $Q$ 2003;7:73-82.

41. Pawson R. Evidence-based policy: a realist perspective. London, UK: Sage Publications Ltd., 2006.

42. Meads C, Nyssen OP, Wong G, et al. Protocol for an HTA report: Does therapeutic writing help people with long-term conditions? Systematic review, realist synthesis and economic modeling. BMJ Open 2014;4:e004377. 